\title{
Load Peak Shaving and Power Smoothing of a Distribution Grid with High Renewable Energy Penetration
}

Ehsan Reihani, Mahdi Motalleb , Reza Ghorbani,and Lyes Saad Saoud ${ }^{1}$

\begin{abstract}
High penetration of renewable energy poses a significant challenge in operation of power system. A potential solution for this problem is utilizing Battery Energy Storage System (BESS). The purpose of this paper is to analyze the effectiveness of BESS ability to peak shave and smooth the load curve of an actual circuit on the island of Maui in Hawaii. The distribution circuit has about $850 \mathrm{~kW}$ of installed rooftop Photo-Voltaic (PV) generation. Higher penetration of $\mathrm{PV}$ increases the concern about the potential impacts on the transmission system. At first, we will present two different methods for load forecasting. Reliable forecasting of daily load is required to effectively utilize the BESS system. We have employed two different methods for load forecasting in order to achieve two main purposes including peak shaving and smoothing. For reaching these goals, two approaches are analyzed. The first approach is utilizing a nonlinear programming method in terms of load shifting and smoothing. The second approach includes a real time control strategy to have smoothing and peak shaving at the same time. As a real case study, these proposed methods have been applied within 108-day data collection period and pros and cons of these methods will be discussed.
\end{abstract}

Keywords: Peak shaving, load smoothing, short term load

\footnotetext{
${ }^{1}$ Ehsan Reihani, Mahdi Motalleb, Reza Ghorbani,and Lyes Saad Saoud are with Renewable Energy Design Laboratory (REDLab) at the Department of Mechanical Engineering, University of Hawaii Manoa, Honolulu, Hawaii, 96822. Phone: +1-808-956-2292, Fax: +1808-956-0767. E-mail: ereihani@hawaii.edu, motalleb@hawaii.edu, rezag@hawaii.edu, saadsaoudl@umbb.dz
}

Preprint submitted to Journal of ${ }^{A} T_{E} X$ Templates

September 7, 2015

(C) 2015. This manuscript version is made available under the Elsevier user license http://www.elsevier.com/open-access/userlicense/1.0/ 
forecasting.

\section{Introduction}

Hawaii Clean Energy Incentive (HCEI) shows a road-map for reducing the use of fossil fuels by achieving $70 \%$ clean energy by 2030 , of which the contribution of efficiency measures and locally generated renewable sources are $30 \%$ and

$540 \%$, respectively. Strong public support for renewable energy in the state [1], has encouraged researchers to investigate new methods to increase the renewable energy penetrations mostly in the form of solar, wind and wave energy. One of the most important issues in renewable integration is intermittency, which creates difficulties in meeting load demand. If a more effective forecasting method could be achieved, these resources could be rendered more reliable thus allowing greater percentage of renewable resources into power grid. BESS is one of the promising solutions to reduce the fluctuations an uncertainties and thus resulting in a more predictable power source. Optimal charging and discharging of BESS, not only reduces the fluctuations of the power output of renewables, but

15 also can be used for peak shaving purposes. In other words, BESS is used to store the excess renewable energy in the off-peak time periods and dump it back into the grid in the high load demand time periods [2]. In this paper, analysis of BESS is undertaken for both purposes. This paper begins with a brief overview of BESS applications, giving context to our research results.

\subsection{Literature review}

State Of Charge (SOC) is a quantity that represents the ratio of available BESS capacity to its fully charged capacity [3]. Li et al. [4] proposed a method in order to smooth the fluctuations caused by PV and wind generation in the power grid using real time SOC based on a control strategy. In the described method, SOC changes around $50 \%$ while alleviating the fluctuations. Ramp rate control was used to smooth the wind power generation [5]. Teleke et al. utilized a predictive control strategy in order to mitigate the wind power fluctuations using BESS [6], so the wind farm can be dispatched in hourly basis. 
A dual BESS designing was proposed by Yao [7] to maximize the energy provides wind power as a short-term dispatchable unit which can be coordinated with other units for active power scheduling. Also, the embedding reliability criteria were considered in the objective function. A dynamic programming based method was used in [8] to design an optimal and cost effective power PV systems. The predictive optimization could save $13 \%$ on the electricity cost for the planned period of study. Additionally, its results could be improved using the higher accurate load forecasting methods.

Enslin investigated PV integration challenges and cites BESS as one of the 40 solutions to deal with the intermittency of the PV resources [9]. There are several mechanisms to make a balance between the generation and the demand which BESS can help the grid operator to reduce the contribution of the spinning reserve in order to deal with the variability. On the other hand, BESS has some problems and limitations such as the limited charging cycles and the high costs.

45 All of these constraints and parameters should be considered to find the optimal solution in a system. Cheng applied different moving average algorithms in order to smooth the PV power curve [10]. Leadbetter used BESS for peak demand shaving of the residential electricity [11]. Based on his method, peak shaving between $42 \%$ and $49 \%$ was reported in 5 regions in Canada except Quebec which was about $28 \%$. The result of his research was the peak shaving system is not appropriate for the houses including electric heating.

Lavrova et al. described an introduction of the smart grid project at Public Service Company of New Mexico (PNM) [12]. The main purpose of their research is to analyze of the cost effectiveness of BESS in terms of smoothing, 55 peak shaving and/or other ancillary services. Profitability level of BESS at utility and residential scale is investigated in [13]. In that research, Tzanetopoulou compared different chemical types of BESS for 6 business cases and as a result, he showed that the molten salt batteries were the most promising type. Additionally, he proved that utility scale BESS had a higher profit potential in 
comparison with the distributed storage.

Another research was done by Pezeshki et al. [14] about the peak shaving and load smoothing using BESS at the community level in Queensland, Australia. They showed about $18 \%$ of the weekly energy cost can be saved. Yang et al. [15] discussed about the capacity of the distributed BESS. They presented

${ }_{65}$ that with the current BESS price, the economic profits might be improved if the multiple functions are considered for the BESS operation. Wang et al. [16] proposed a load forecasting method which was appropriate for the residential storage controller. Their purpose was peak shaving and electricity cost minimization. The result of the proposed method was saving the cost about $80 \%$ with respecting to the baseline algorithm. Hoiles [17] worked on nonparametric forecasting the demand to increase the grid reliability based on Afriats theorem.

In order to solve the forecasting problems in the power systems, different computational intelligence techniques have been widely used, such as Artificial Neural Networks (ANN) [18, 19, 20, 21, 22], fuzzy-logic approach [23], and 75 genetic algorithm [24, 25]. Anbazhagan [26] did price forecasting for day-ahead via recurrent neural networks. A model for the wind forecasting and wind generation was proposed by Aquino et al., using ANN [27]. Combination of the load and wind power forecasting was done by Quan et al. via neural networkbased prediction intervals [19].

80 Yona et al. [28] described a method for insolation prediction with fuzzy and then added ANN for long term ahead corrected prediction of photo-voltaic outputs. Kurbatsky et al. [29] applied two stages of adaptive neural network to have a short-time forecast of power system parameters. Lee worked on the short-term forecasting the wind power via Gaussian processes and ANN [20]. Saez et al. [30] developed a fuzzy prediction interval model for a microgrid to forecast the renewable resources and loads.

\subsection{Contribution of the proposed method}

The main contribution of this paper is simultaneous application of peak shaving and smoothing on the load curve of a distribution grid. The focus of 
this paper is proposing the algorithms and methods to achieve this goal so that the transmission system is relieved from the aggregate fluctuations of rooftop PV generations. The second section of this paper covers the system model and the problem statement. A parallel forecasting method has been developed using Complex-Valued Neural Networks (CVNN) in which the load data for the next

9524 hours is obtained. Load smoothing needs a fairly accurate load forecast and thus a new approach is proposed to predict the load value for 20 minutes ahead. The fluctuations on the load curve are removed by BESS while trying to keep the BESS SOC changes at minimum level. Peak shaving is performed along with smoothing to make the best use of the BESS capacity. In section 2 the circuit is presented and more information about the interconnection of BESS and the distribution system is provided. In this paper two approaches are used in order to achieve the mentioned goals. The first approach uses nonlinear programming and the second method utilizes a simple algorithm to control the SOC of BESS in real time. Both methods require forecasting of the load curve and thus section 3 discusses forecasting in detail which comes subsequently.

Two heuristic approaches are presented in section 4. The first method uses a forecast load curve and yields a SOC trajectory for peak shaving and also smoothing. By injecting/absorbing the power determined by SOC trajectory, peak shaving and/or smoothing goals are reached. Next, a simple algorithm which makes use of real time control of BESS SOC is covered. This method benefits from a better forecasting method and is easy to implement. It also includes some user-interactive parameters which can be set by the operator or fed from another power system application. Section 5 includes the results of the simulations. Finally, conclusion and the future works are described in section 6 .

\section{System Model and Problem Statement}

The model of the system has been defined in an island where several generators meet the load demand. Fig.1 shows the information flow diagram of 
the island model which several BESS units manage the distribution load. En-

ergy Management System (EMS) receives the measured data from BESS units , generators and other controllable devices via Supervisory Control And Data Acquisition (SCADA). Hybrid Forecast And Load Commitment (HFALC) program is amended to EMS to manage the BESS operation.

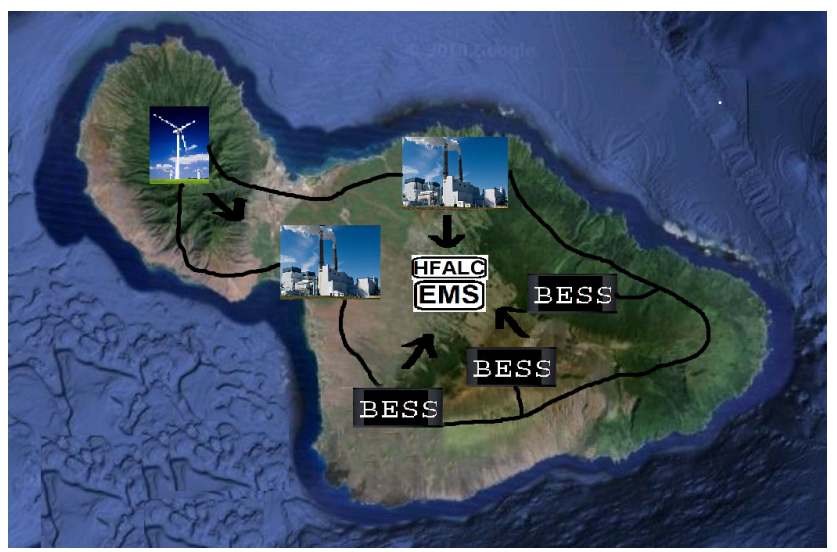

Figure 1: Overall information flow in system model

Fig.2 describes HFALC utilizing CVNN and series-parallel load forecasting data in order to optimize the BESS performance. The information flows from EMS to the forecasting algorithms and then the optimized command is sent back to EMS. Additionally, the user preference is provided for the optimization and forecast algorithms. After finding the improved operation points, the required data is sent back to EMS for more processing. Then the required commands are sent to the control units from EMS. More details about the HFALC are described in the next sections.

Fig.3 demonstrates the electrical circuit of the BESS in the distribution grid. The capacity of the whole grid is $300 \mathrm{MW}$ generation which the contribution of the wind farms is $40 \mathrm{MW}$. An $1100 \mathrm{kWh} / 1 \mathrm{MW}$ BESS is connected to a power converter which its output voltage is increased with a step-up transformer. The power of the BESS flows to a distribution circuit which has about $850 \mathrm{~kW}$ rooftop PV generation. The distributed PV generation causes some fluctuations in the 


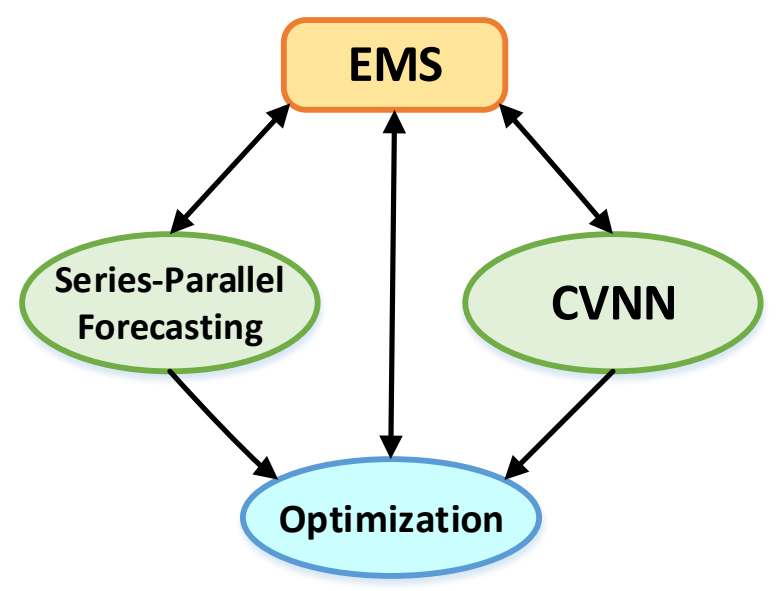

Figure 2: HFALC unit interaction with EMS

power curve. This circuit is also fed from a distribution transformer with the ratio of $69 \mathrm{kV} / 12.47 \mathrm{kV}$.

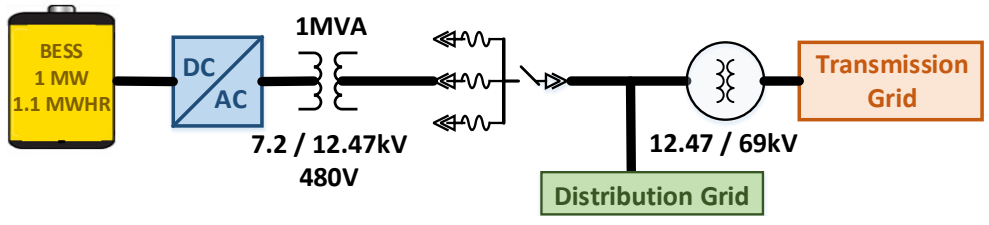

Figure 3: BESS connection to the distribution grid system

SOC of the BESS and other health parameters are transmitted via SCADA system to the dispatch room where an operator can control the BESS operation using a GUI. Power flow in the distribution transformer is also measured by SCADA which is available via a database in the utility. The purpose of the BESS control is to mitigate the variability of the power of the distribution transformer and also reduce the amount of committed generating units to meet the demand. This is possible with charging the BESS by excess renewable energy and dumping back the stored energy of the BESS to the grid in the peak load time, so the generation units which are not environmentally friendly 
can be replaced easily by the BESS in order to meet the demand especially in the peak load time. Additionally, BESS provides smoothing function in midday when the load intermittency is pretty high due to distributed PV generation of the distribution circuit. The next section covers the forecasting methods which have been used in the solution approaches for the mentioned objectives.

\section{Proposed forecasting methods}

In order to apply the optimization algorithms for peak shaving and smoothing, we have to go through load forecasting method. Any forecast deviation from actual values for the next 20 minutes ahead can be easily compensated with rapid startup generators [31]. Since the battery capacity is limited, accurate forecasting algorithms improve operation of BESS more efficiently. The day ahead load forecasting is required for peak shaving purpose while 20 minutes ahead forecast is mostly effective for smoothing purpose. The two methods used in forecasting are CVNN (called parallel) and series-parallel.

\subsection{Complex-Valued Neural Networks}

The major difference between ANN and CVNN is the nature of using parameters. The real numbers are part of the complex ones, so CVNN is as an extension of ANN.

Fig. 4 shows the CVNN's architecture including the input, hidden, and output layers. The input layer includes two parts: the input data $(v)$ and the previous output data $(y)$.

If the input vector $\left(X_{m}\right)$ and the weights (i.e., $W_{n 0}^{1}, W_{l 0}^{2}, W_{n m}^{1}, W_{l n}^{2}$, etc.) and the activations function $\left(f_{c}\right)$ have real values, the network is normal ANN, otherwise, with the complex values of the mentioned parameters, the network is CVNN.

Equation (1) shows the value of the $l^{\text {th }}$ output of the network (forecast 175 value):

$$
\hat{y}_{l}(k)=f_{c}\left(W_{l 0}^{2}+\sum_{n} W_{l n}^{2} H_{n}\right)
$$




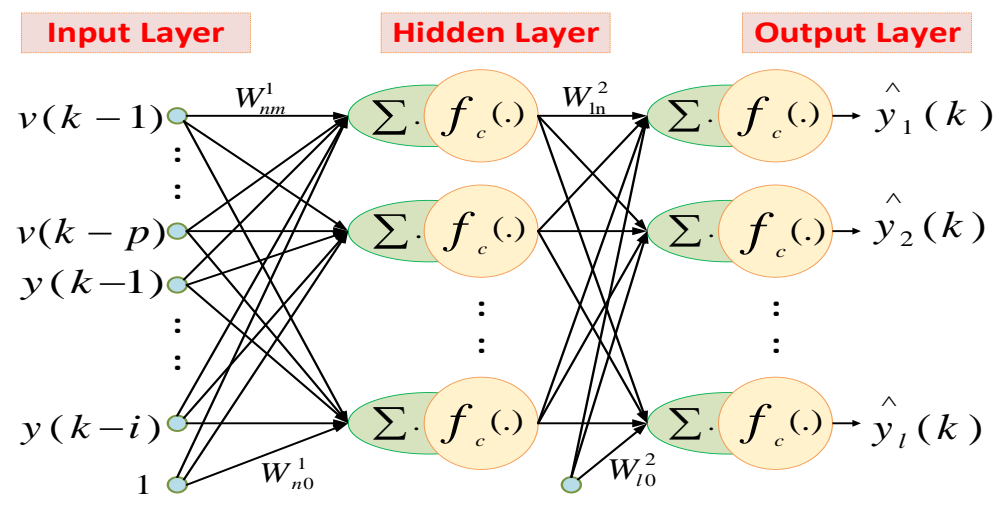

Figure 4: Architecture of CVNN including the nodes, layers, and weights

and the output of $n^{\text {th }}$ hidden neuron is given as:

$$
H_{n}=f_{c}\left(W_{n 0}^{1}+\sum_{m} W_{n m}^{1} X_{m}\right)
$$

where $X_{m}=[v(k-1), v(k-2), \ldots, v(k-p), y(k-1), y(k-2), \ldots, y(k-i)]$, which includes $m$ complex valued inputs $(m=i+p)$. The whole values of $m$ $, n, i, l$, and $p$ are positive integers $[32,33,22]$. In this paper, the split sigmoid function has been taken for the activation function as follows:

$$
f_{c}(z)=\frac{1}{1+e^{-\operatorname{Re}(z)}}+j \frac{1}{1+e^{-\operatorname{Im}(z)}},
$$

where $z=x+j y$, with $j=\sqrt{-1}$. Using the split sigmoid function instead of non-split, avoids the problem of function's singularity. After applying the complex back-propagation algorithm and calculation of the weight values $\left(\triangle W_{l 0}^{2}, \triangle W_{l n}^{2}, \triangle W_{n 0}^{1}\right.$, and $\left.\triangle W_{n m}^{1}\right)$, the error can be calculated in the following 185 equation:

$$
e_{l}(k)=y_{l}(k)-\hat{y}_{l}(k)
$$

where $e_{l}(k)$ is the error between the $l^{\text {th }}$ actual output $y_{l}(k)$ and forecast output $\hat{y}_{l}(k)[33,22]$.

\subsection{Series-Parallel forecasting}

In this method, in addition to the historical load data, past data of each day 190 is used. The past data spans from 20 minutes ago until the present moment. 
Moreover, the load data point calculated by parallel forecasting (CVNN) is also required. As a result, a set of data is prepared which includes the past data and a point from future forecast. A spline interpolation is fit into this set of data. In the next step, the new points produced by spline interpolation, are averaged. The generated point comprises one component of the final forecast value. This point is compared with the actual value when the future time spot is reached.

Then, a weighted value of this error is added to the averaged value and makes the final forecast value. Apparently, in the first forecast point, this error does not exist and the above steps work for forecasting after the first point. This process is refereed as PI control shown in Fig. 5 since it mimics the Proportional Integral (PI) control mechanism. The 20-minute interval can be changed to suit user preference. At this point, the required future behavior of load curve is known to some certainty. This information is employed for optimal control of BESS to accomplish the defined objectives.

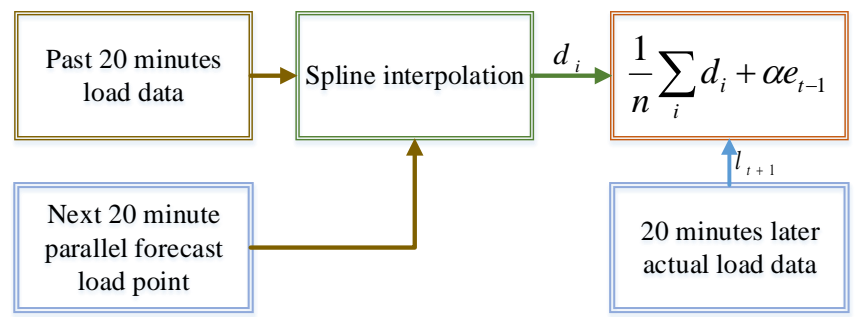

Figure 5: Flowchart of series-parallel load forecasting method

\section{Proposed approaches}

This section includes two proposed approaches which both of them are based on the mentioned forecasting methods.

\subsection{Approach $I$}

In approach one, peak shaving and smoothing objectives are handled separately. First of all, a SOC trajectory is found by solving the peak shaving 
problem and BESS power is determined to follow the obtained trajectory. Next, fluctuations in the midday are curbed while sticking to the planned SOC trajectory as much as possible. In the next subsection, the peak shaving problem is explained and SOC trajectory for next 24 hours is obtained.

\subsubsection{Peak Shaving}

For peak shaving, it is desired to charge BESS in early morning when there is excess renewable energy production usually from wind turbines and discharge it to replace the expensive units which pick up the peak load. In other words, BESS power flow tries to flatten the load curve considering imposed constraints.

220 Therefore, cost function is defined as minimization of the total load deviation from a straight line. This line is determined based on the forecast load curve already obtained. The total load consists of forecast load and BESS power. In other words, BESS tries to maintain the load curve as straight line knowing its capacity. Based on this explanation, the mathematical objective function is written as follows:

$$
\min \left(\sum_{t=1}^{N}[L(t)+P(t)]^{2}\right)
$$

where $N$ is the number of time steps in forecasting horizon. Parameters $L(t)$ and $P(t)$ stand for forecast load and BESS power at time stage $t$. The above equation can be derived from a different perspective as well. Suppose objective function at time step $t, J(t)$, is defined as the total load flowing the distribution transformer times a time variant coefficient:

$$
J(t)=(L(t)+P(t)) W(t)
$$

where $W(t)$ represents load coefficient at time step $t$. In order to penalize the high load flowing the distribution transformer, $W(t)$ is chosen as square of overall power flow:

$$
W(t)=(L(t)+P(t))^{2}
$$


Plugging (7) into (6), taking first derivative and summing up over the horizon

results in equation (5). SOC constraints are described as:

$$
\begin{aligned}
& P(t)>\frac{1}{\Delta t}\left[S O C_{\text {min }} E_{t o t}-E(t)\right] \\
& P(t)<\frac{1}{\Delta t}\left[S O C_{\max } E_{t o t}-E(t)\right]
\end{aligned}
$$

SOC of BESS is updated by:

$$
S O C(t)=S O C(t-1)+P(t) \Delta t / E_{t o t}
$$

In the above equations, $S O C_{\min }$ and $S O C_{\max }$ denote the minimum and maximum allowable SOC. $E_{\text {tot }}$ and $E(t)$ show the capacity of BESS and stored energy at time step $t$ respectively. $\Delta t$ represents the planning time resolution. Equation (5) is a routine constrained nonlinear programming which can be tackled with regular solvers in Matlab ${ }^{\circledR}$ or other commercial or non-commercial programs such as GAMS $^{\circledR}$ and AMPL ${ }^{\circledR}$. Solving cost function (5) gives the BESS active power flow at each time step and also the resulting SOC.

\subsubsection{Smoothing}

245

Smoothing is an add-on function amended to peak shaving problem to harness the stochastic variability in the load curve. The underlying concept is the same as peak shaving. That is, at each time interval, a level is determined and BESS tries to keep this straight total line. In other words, BESS is optimized to shave the peak while maintaining the smoothing level during each interval.

This level should be determined in a way that SOC deviation is minimum at the end of each time interval. Therefore, smoothing level is defined as the average of current and forecast load.

Consequently, the amount of BESS charge and discharge should be almost equal and thus SOC deviation is kept at minimum level. The more forecast is equal to the actual value, less SOC deviation occurs at the end of each period. The error of SOC, $S O C_{e}$, at time $t$ is defined as:

$$
S O C_{e}(t)=\left(S O C(t-1)+\left(P(t)+P_{x}(t)\right) \Delta t / E_{t o t}+S O C(t)\right)^{2}
$$


In the above equation, $P_{x}(t)$ denotes BESS supplemental power provided for SOC correction. Smoothing error, $S M_{e}$, at time $t$ is expressed as:

$$
S M_{e}(t)=\left(\left(L(t)+P(t)+P_{x}(t)-S L(t)\right)^{2}\right.
$$

where smoothing level is a combination of following terms:

$$
S L(t)=0.5 \times L(t)+.5 \times f(t+\Delta t)+S O C(t+\Delta t)-S O C(t)
$$

The two first components comprise the average of current and series-paralell forecast value. The remaining terms, which represent SOC deviation from a preplanned point, are added to make up for the SOC deviation. In addition to that, $f$ which denotes 20 minutes ahead load forecast, is responsible for calculating the smoothing level. Smoothing level is updated at the end of each period. Total cost is weighted sum of SOC and smoothing error:

$$
\text { Cost }=\sum_{t=0}^{N}\left(\alpha S O C_{e}(t)+\beta S M_{e}(t)\right)
$$

where $\alpha$ and $\beta$ are coefficients for each component of cost function. Optimal BESS power is obtained by minimizing the cost function:

$$
P_{x}=\arg \min _{P_{x}} \text { Cost }
$$

After finding $P_{x}$, BESS power is updated by $P_{x}$ :

$$
P(t)=P(t)+P_{x}(t)
$$

SOC is also calculated by equation(10) accordingly.

\subsection{Approach II}

In the second method, BESS operation is divided into three time sections namely, charging, smoothing and discharging. However, smoothing is embedded in charging and discharging functions serving peak shaving goal. In spite of some fluctuations in SOC caused by smoothing implementation, BESS is kept almost full during smoothing period to be able to dump back stored energy in the peak 
shaving period. The goal of smoothing which is present in all time periods is to filter out the load stochastic variability.

The smoothing level in approach II changes to an inclined line whose slope is based on the startup time of rapid start generators such gas turbines and diesel generators. Aside from that, fast generators can make up any load discrepancy within allowed time. Moreover, BESS removes the fluctuations around the line while maintaining the SOC level. It is assumed that BESS is empty with SOC of about $20 \%$ and needs to be charged until early morning. Usually, there is higher generated power form windfarm before sunrise and the load is pretty much low at the same time.

As a result, some of the generated power has to be curtailed. Therefore, it is preferred to charge BESS with the curtailed power. Even if there is no generated power from windfarm, BESS can be charged from base load generators. Apparently, the stored energy will meet the peak demand at early night which would be otherwise met with gas turbines and diesel generators. In order to come up with line equation for the inclined reference power line, two points are required. The first point is the current load and the next point comes from the 20 minutes ahead forecast load. BESS is counteracting the load around the defined line so that total load is an inclined line regardless of what is happening 295 in the circuit.

Regardless of whether the load fluctuations are from PV or abnormal load value at peak and off-peak time periods, BESS sticks to the defined power line. Consequently, the power line at each time step $t_{k}, P_{L}\left(t_{k}\right)$, is written below:

$$
P_{L}\left(t_{k}\right)=(P(t)+L(t))+m\left(t_{k}-t\right)
$$

where $m$ is the slope of the power line and is calculated by:

$$
m=\frac{f(t+\Delta t)+g(S O C(t))-(P(t)+L(t))}{\Delta t}
$$

The load forecast for next time step is presented by $f(t+\Delta t)$. To add power system operator preference, slope of the power line is updated by $g(S O C(t))$. For instance, the operator observes an increase in wind power generation and 
thus updates the slope accordingly. For charging, the slope needs to be higher than bare one and thus the function is given a leverage. Charging continues until SOC reaches almost $80 \%$ to be considered full. Apparently, for discharging the reverse action should be followed. The smoothing function begins when BESS is almost full and continues when the peak load level is detected. BESS power flow is determined like (17) except SOC deviation is corrected in the slope of smoothing:

$$
\begin{gathered}
P_{L}\left(t_{k}\right)=(P(t)+L(t))+(m-d S O C(t))\left(\left(t_{k}-t\right)\right) \\
m=\frac{f(t+\Delta t)+g(S O C(t))-(P(t)+L(t))}{\Delta t}
\end{gathered}
$$

where $d S O C$ is given as:

$$
d S O C=S O C(t)-0.8
$$

Besides correction embedded in $m, g(S O C(t))$ can be also utilized for the same purpose. As soon as peak load level is measured, BESS starts to discharge the stored energy and will continue until it is almost empty, i.e., SOC reaches nearly $31520 \%$.

\section{Simulation results}

The proposed approaches are used to control BESS power flow using 108 days of actual distribution system active power data. At the first, results of forecast methods are given and compared with actual load curve. Then, impact of BESS on peak shaving and smoothing of load curve for both approaches is investigated.

\subsection{Forecasting results}

CVNN and series-parallel methods are applied on the historical load data for coming up with an approximate behavior of load curve in the future. The data and the algorithm are processed in Matlab ${ }^{\circledR}$ environment. In CVNN method, 107 days of historical data are used to forecast the 108th day. The forecast load 
curve obtained from set of equation 1-4 along with actual load data is depicted in Fig. 6. The forecast results by CVNN have been shown in two different forms including the real forecast data and the smoothed forecast data by Kalman Filter. Kalman Filter [34] is a mathematical process in order to minimize the mean of the squared error. .
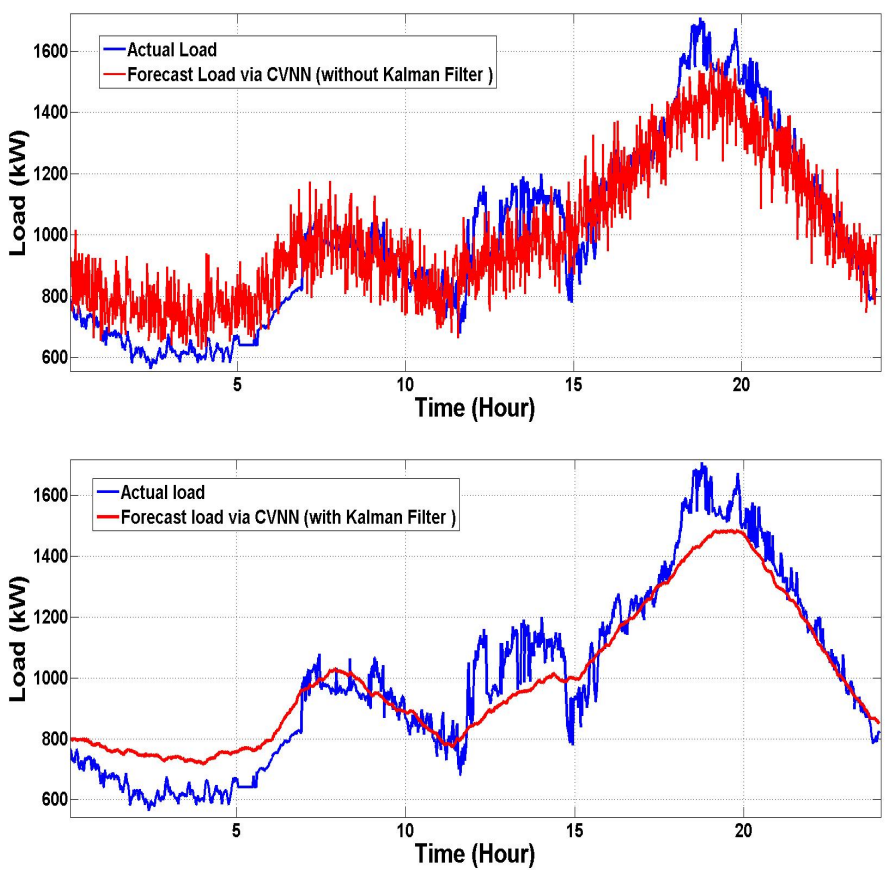

Figure 6: CVNN forecasting method (with and without Kalman Filter) along with the actual load data

When the amount of the fluctuation is high, especially in midday, the actual and forecast load curves differ significantly while in other parts of the day, the two curves are fairly close to each other. Series-parallel method is employed to forecast load data for the next 20 minutes. The forecast result accompanied with actual data sampled at 20-minute interval is depicted in Fig. 7. 


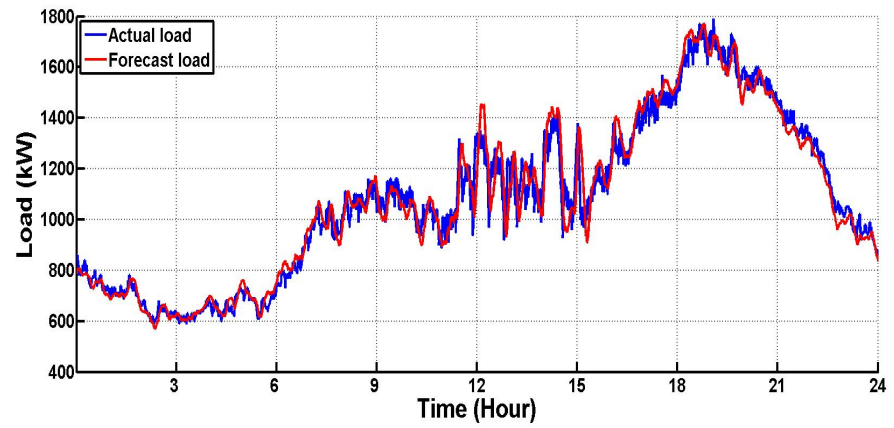

Figure 7: Series-parallel method for 20 minutes look ahead forecast accompanied with actual load data

\subsection{Approach I results}

In the first simulation, peak shaving is performed on the 104th day using equation 5-9 where the load is obtained using CVNN forecast method expressed in equations 1-4. Pure peak shaving method tries to keep the load curve as flat as possible based on the predicted curve and SOC constraints. Solving equations 1-4 gives the target SOC trajectory shown in Fig. 8 which BESS will follow by absorbing or injecting active power to the distribution circuit. Looking at the forecast load in Fig. 6, it is easy to see how SOC trajectory in Fig. 8 is obtained.

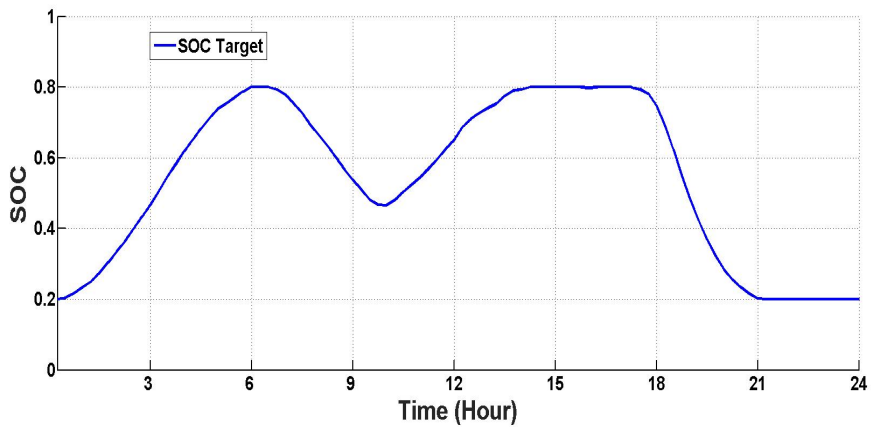

Figure 8: SOC trajectory obtained from approach I

As shown in Fig. 8, SOC is increasing to shift the load curve by charging from midnight until 6 a.m. As the concavity of the load curve changes around 
6.a.m, BESS discharges some of the stored energy until about 10 a.m. to lower the load curve. After the local minimum in SOC trajectory which happens around 10 a.m., BESS charges up to 2 p.m. to increase the load which is a little below the flat line around $800 \mathrm{~kW}$. After 2 p.m. until 6 p.m., BESS keeps the stored energy and starts to discharge to shave the peak. The compensated load flowing through the transformer is depicted in Fig. 9.

As it can be seen in Fig. 9, the corrected load is almost flat until 8 a.m. but will differ a little due to error in forecasting. Moreover, since the forecast and actual load are different significantly around noon, the corrected load is even higher than actual load. BESS discharges the stored energy in the evening and flattens the load curve.

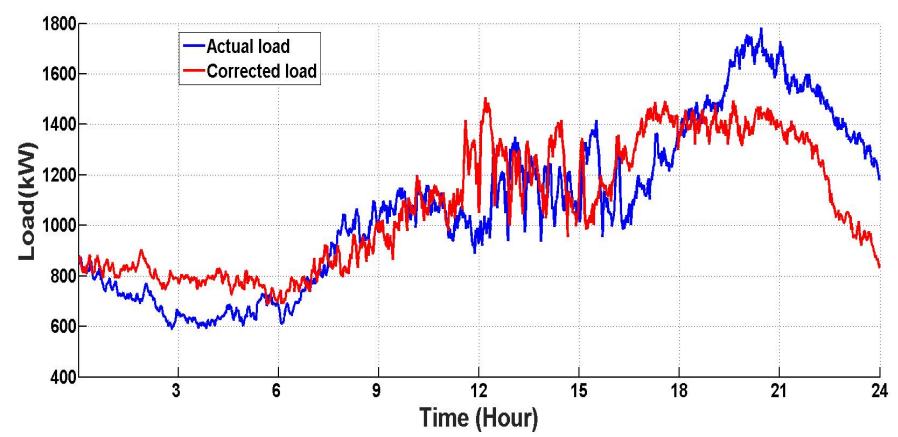

Figure 9: Circuit load corrected by BESS operation calculated by approach I

\subsection{Approach II results}

The second approach benefits from real time control of BESS power using series-parallel forecast. BESS follows the power trajectory which is an inclined line between the current load and 20 minutes load forecast. The function $g(S O C(t))$ in equation (20) is chosen as a simple constant $150 \mathrm{~kW}$ for charging, 0 for smoothing and $-(S O C(t-1) * 390)$ for discharging period. Therefore, BESS will charge following the line whose slope is defined by a leverage of 150 $\mathrm{kW}$ above the forecast value. In the smoothing period, BESS tries to decrease the fluctuations by creating a power line where the forecast value is kept unchanged. The slope of discharging line depends on the available energy. That 
is, as the stored energy reduces, the slope of line decrease and thus discharging functions approaches the smoothing function. The obtained SOC by real time control in approach II has been shown in Fig.10.

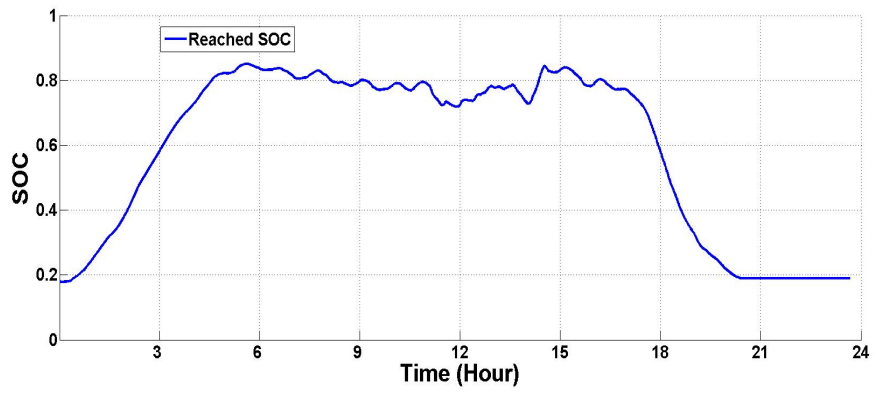

Figure 10: BESS SOC obtained by real time control described in approach II

As shown in these figures, this method does not suffer from the shortcomings of previous approaches in smoothing period. In other words, using real time control, BESS tries to smooth the fluctuations with minimal change in SOC. As a result, BESS remains almost full in the beginning of discharging period and dumps back stored energy to shave the peak load. It is also clear from Fig. 11 that BESS has removed the fluctuations in the load and the corrected load has changed to piecewise linear curve. In short, BESS has removed the variability of load curve without much affecting SOC and has shifted the load to make use of renewables generated in early morning for meeting the demand at night.

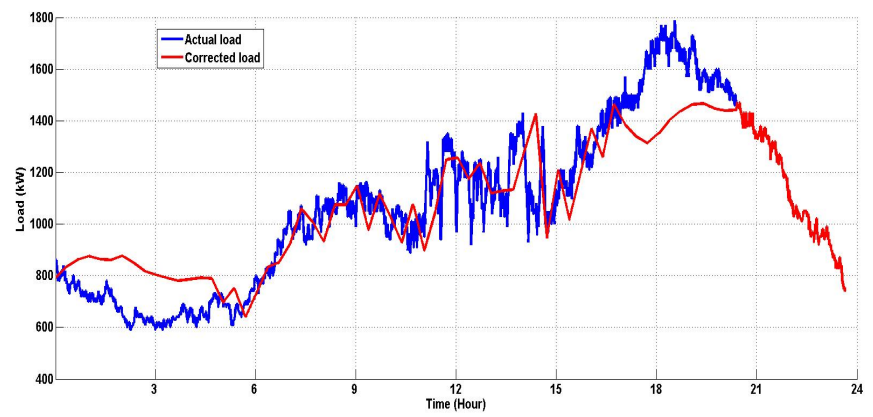

Figure 11: Actual and compensated load using the algorithm explained in approach II 


\section{Conclusion}

380 with actual load values. Load data points are obtained for next 24 hours using CVNN method. CVNN method does not perform very well during high PV fluctuations. The second forecasting method called series-parallel forecasting method uses the past data of forecast say and can predict 20 minutes ahead load data point with fairly high accuracy. The forecast load values are used to perform peak shaving and smoothing on the load curve of distribution grid. For peak shaving purpose, BESS follows a SOC trajectory which is obtained by an optimization algorithm using CVNN forecast approach. This method suffers from inaccurate forecast load which leads to unsuitable charge/discharge function is added to reduce the fluctuation while performing the peak shaving task. This method also relies on series-parallel forecasting method while adds smoothing to the shaved peak load curve. This method takes advantage of fairly accurate load forecast and performs both tasks. The smoothing approach not only shifts the load but also damps the load stochastic fluctuations mostly caused by PV generation. There are some user interactive parameters which can be coordinated with other variables in power system for an optimal operation. The series-parallel method benefits from the real time data of the circuit load and weather forecast may help get a better load forecast value for the next time step. With more deployment of BESS, the amount of variability in transmission system decreases and this leads to more integration of renewables, especially rooftop PV generations.

\section{References}

[1] B. DeBaryshe, I. Stern, Public attitudes about renewable energy in Hawaii 405 (2014). 
[2] S. Sepasi, R. Ghorbani, B. Liaw, Improved extended kalman filter for state of charge estimation of battery pack, J. Power Source 255 (1) (2014) 368376.

[3] S. Sepasi, R. Ghorbani, B. Liaw, A novel on-board state-of-charge estimation method for aged li-ion batteries based on model adaptive extended kalman filter, J. Power Source 245 (1) (2014) 337-344.

[4] X. Li, D. Hui, X. Lai, Battery energy storage station (bess)-based smoothing control of photovoltaic (pv) and wind power generation fluctuations, IEEE Trans. Sustain. Energy 4 (2) (2013) 646-473.

[5] M. C. Such, C. Hill, Battery energy storage and wind energy integrated into the smart grid, in: Innovative Smart Grid Technologies, Vol. 3, IEEE, 2012, pp. 1-4.

[6] S. Teleke, M. Baran, S. Bhattacharya, A. Huang, Optimal control of battery energy storage for wind farm dispatching, IEEE Trans. Energy Convers. 25 (3) (2010) 787-794.

[7] D. Yao, S. Choi, K. Tseng, T. Lie, Determination of short-term power dispatch schedule for a wind farm incorporated with dual-battery energy storage scheme, IEEE Trans. Sustain. Energy 3 (1) (2012) 74-84.

[8] Y. Riffonneau, S. Bacha, F. Barruel, S. Ploix, Optimal power flow management for grid connected pv systems with batteries, IEEE Trans. Sustain. Energy. 2 (3) (2011) 309-320.

[9] J. Enslin, Integration of photovoltaic solar power the quest towards dispatchability, IEEE Instrumentation and Measurement Magazine 17 (2) (2014) 21-26.

[10] F. Cheng, The management of a utility-scale battery storage system for renewable energy applications, Master's thesis, The University of New Mexico, Albuquerque, New Mexico (May 2012). 
[11] J. C. R. Leadbetter, Residential battery energy storage systems for renewable energy integration and peak shaving, Master's thesis, Dalhousie University (2012).

[12] O. Lavrova, et al., Analysis of Battery Storage Utilization for Load Shifting and Peak Smoothing on a Distribution Feeder in New Mexico, in: Innovative Smart Grid Technologies (ISGT), IEEE, 2012, pp. 1-6.

[13] C. Tzanetopoulou, Techno-economic assessment of battery solutions for solar plants and distribution system operation, Master's thesis, Swiss Federal Institute of Technology, EEH Power Systems Laboratory, ETH Zurich (July 2014).

[14] H. Pezeshki, P. Wolfs, G. Ledwich, A model predictive approach for community battery energy storage system optimization, in: PES General Meeting, IEEE, 2014, pp. 1-5.

[15] Y. Yang, et al., Sizing strategy of distributed battery storage system with high penetration of photovoltaic for voltage regulation and peak load shaving, IEEE Trans. Smart Grid 5 (2) (2014) 982-991.

[16] Y. Wang, X. Lin, M. Pedram, Adaptive control for energy storage systems in households with photovoltaic modules, IEEE Trans. Smart Grid 5 (2) (2014) 992-1001.

[17] W. Hoiles, V. Krishnamurthy, Nonparametric demand forecasting and detection of energy aware consumers, IEEE Transactions on Smart Grid 6 (2) (2015) 695-704.

[18] S. Kalogirou, E. Mathioulakis, V. Belessiotis, Artificial neural networks for the performance prediction of large solar systems, Renewable Energy 63 (2014) 9097.

[19] H. Quan, D. Srinivasan, A. Khosravi, Short-term load and wind power forecasting using neural network-based prediction intervals, IEEE Transactions on Neural Networks and Learning Systems 25 (2) (2014) 303-315. 
[20] D. Lee, R. Baldick, Short-term wind power ensemble prediction based on gaussian processes and neural networks, IEEE Transactions on Smart Grid 5 (1) (2014) 501-510.

[21] A. Vaz, B. Elsinga, W. van Sark, M. Brito, An artificial neural network to assess the impact of neighbouring photovoltaic systems in power forecasting in utrecht, the netherlands, Renewable Energy 85 (2016) 631-641.

[22] M. Motalleb, S. Sreedarsan, R. Ghorbani, Heuristic power grid reliability improvement under system contingency, IEEE Transactions on Power Systems (Under Review).

[23] S. Chen, H. Gooi, M. Wang, Solar radiation forecast based on fuzzy logic and neural networks, Renewable Energy 60 (2013) 195-201.

[24] V. Kurbatsky, N. Tomin, D. Sidorov, V. Spiryaev, Hybrid genetic algorithms for forecasting power systems state variables, in: IEEE Grenoble PowerTech, IEEE, 2013.

475 [25] H. Liu, H. Tian, X. Liang, Y. Li, New wind speed forecasting approaches using fast ensemble empirical model decomposition, genetic algorithm, mind evolutionary algorithm and artificial neural networks, Renewable Energy 83 (2015) 1066-1075.

[26] S. Anbazhagan, N. Kumarappan, Day-ahead deregulated electricity market price forecasting using recurrent neural network, IEEE Systems Journal 7 (4) (2013) 866-872.

[27] R. de Aquino, H. Gouveia, M. Lira, A. Ferreira, O. Neto, M. Carvalho, Wind forecasting and wind power generation: Looking for the best model based on artificial intelligence, in: The International Joint Conference on Neural Networks (IJCNN), IEEE, 2012.

[28] A. Yona, T. Senjyu, T. Funabashi, C.-H. Kim, Determination method of insolation prediction with fuzzy and applying neural network for long-term 
ahead pv power output correction, IEEE Transactions on Sustainable Energy 4 (2) (2013) 527-533.

490

[29] V. Kurbatsky, N. Tomin, D. Sidorov, V. Spiryaev, Application of two stages adaptive neural network approach for short-term forecast of electric power systems, in: 10th Environment and Electrical Engineering International Conference(EEEIC), IEEE, 2011.

[30] D. Saez, F. Avila, D. Olivares, C. Canizares, L. Marin, Fuzzy prediction interval models for forecasting renewable resources and loads in microgrids, IEEE Transactions on Smart Grid 6 (2) (2015) 548-556.

[31] W. Corporation, Combustion engine vs gas turbine: startup time (2015). URL http://www.wartsila.com/en/power-plants/learning-center/ combustion-engine-vs-gas-turbine-startup-time

[32] A. Hirose, Complex-Valued Neural Networks: Advances and Applications, IEEE Press Series on Computational Intelligence, 2013.

[33] L. S. Saoud, F. Rahmoune, V. Tourtchine, K. Baddari, Complex-valued forecasting of the global solar irradiation, Journal of Renewable and Sustainable Energy 5 (2013) 043124:1-21.

[34] O. B. Shukur, M. H. Lee, Daily wind speed forecasting through hybrid kf-ann model based on arima, Renewable Energy 76 (2015) 637647. 